\title{
NONLINEAR RESPONSE OF A CONCENTRATED FERROFLUID TO A LOW-FREQUENCY MAGNETIC FIELD
}

\author{
A.A. Kuznetsov, A.V. Lebedev, A.F. Pshenichnikov \\ Institute of Continuous Media Mechanics of the Ural Branch, RAS, \\ 1 Ac. Korolev str., 614013 Perm, Russia
}

Magnetic response of a concentrated polydisperse ferrofluid to a strong linearly polarized applied field is investigated both experimentally and theoretically. It is shown that in a wide range of particle concentrations and field amplitudes the second-order modified mean-field theory by Ivanov and Kuznetsova [2] well describes the experimentally observed magnetization spectra if the field frequency is low enough and internal relaxation processes in ferrofluid can be neglected.

Introduction. Interactions between dispersed nanoparticles have a significant impact on the properties of concentrated ferrofluids [1]. The influence of the interactions (primarily, steric and dipole-dipole ones) on the magnetization curve and initial static susceptibility of ferrofluids has been discussed extensively [2-5]. Much less attention has been paid to the behavior of concentrated nanoparticle suspensions in nonstationary magnetic fields [6]. However, the latter problem is of great practical importance since the dynamic magnetic response is a basis for many biomedical applications [7] and characterization methods [8] of ferrofluids.

The present work is devoted to a theoretical and experimental investigation of how the interparticle interactions influence the behavior of dense ferrofluids in a strong alternating magnetic field.

1. Theory. Let us consider an elongated cylindrical cavity filled with a ferrofluid. A linearly polarized magnetic field $H(t)=H_{0} \cos \omega t$ acts along the cavity long axis. The frequency $\omega$ is considered low enough so that $\omega \tau \ll 1$, where $\tau$ is the characteristic relaxation time of the particle magnetic moment. If this condition is fulfilled, the magnetization $M$ of the system at an arbitrary instant of time can be found as

$$
M(t)=M_{\mathrm{eq}}(H(t))
$$

where $M_{\mathrm{eq}}=M_{\mathrm{eq}}(H)$ is the equilibrium magnetization curve. The latter can be determined in the framework of the second-order modified mean-field theory (MMFT2) [2]:

$$
\begin{gathered}
M_{\mathrm{eq}}(H)=M_{\mathrm{L}}\left(H_{\mathrm{mf}}\right), \quad M_{\mathrm{L}}(H)=n \int_{0}^{\infty} m(x) f(x) L\left(\frac{\mu_{0} m(x) H}{k_{\mathrm{B}} T}\right) \mathrm{d} x, \\
H_{\mathrm{mf}}=H+\frac{M_{\mathrm{L}}(H)}{3}+\frac{M_{\mathrm{L}}(H)}{144} \frac{\mathrm{d} M_{\mathrm{L}}(H)}{\mathrm{d} H}, \quad L(\xi)=\operatorname{coth} \xi-\xi^{-1}, \\
M_{\mathrm{eq}}(H \rightarrow 0)=\chi H, \quad \chi=\chi_{\mathrm{L}}\left(1+\chi_{\mathrm{L}} / 3+\chi^{2} / 144\right), \quad \chi_{\mathrm{L}}=\mu_{0}\left\langle m^{2}\right\rangle n / 3 k_{\mathrm{B}} T,
\end{gathered}
$$

where $n$ is the particle concentration, $f(x)$ is the distribution of the particle magnetic diameters $x, m(x)=\pi M_{\mathrm{s}} x^{3} / 6$ is the particle magnetic moment, $M_{\mathrm{s}}$ is the saturation magnetization of the particle material, $k_{\mathrm{B}}$ is the Boltzmann constant, $T$ is the temperature, $\chi$ is the ferrofluid initial static susceptibility, $M_{\mathrm{L}}=M_{\mathrm{L}}(H)$ is the magnetization curve in the Langevin approximation (i.e. without interactions), $\chi_{\mathrm{L}}$ is the initial susceptibility in the Langevin approximation, $L(\xi)$ is the Langevin function, $\left\langle m^{2}\right\rangle=\int_{0}^{\infty} m^{2}(x) f(x) \mathrm{d} x$. 


\section{A.A. Kuznetsov, A.V. Lebedev, A.F. Pshenichnikov}

The choice of MMFT2 is due to the fact that it accurately describes magnetization curves of the most real ferrofluids in a wide range of concentrations and dipole-dipole interaction energies [5]. The only exceptions are ferrofluids with $\chi \gtrsim 100$ [4]. If $H_{0}, \omega, f(x), T, M_{\mathrm{s}}$ and $\chi$ are given, one can determine $M(t)$ from Eqs. (1), (2). Then it can be expanded in a Fourier series

$$
M(t)=\sum_{k=1}^{\infty} A_{k} \cos \left(k \omega t+\theta_{k}\right),
$$

where $A_{k}$ is the amplitude of the $k$-th harmonic and $\theta_{k}$ is its initial phase angle. In the weak-field limit, magnetization is directly proportional to the field, and the right-hand side of Eq. (3) contains only the term with $k=1$. At nonvanishing amplitudes, higher harmonics will appear due to the nonlinearity of the magnetization curve. The contribution of the $k$-th harmonic to the total system response will be characterized here by the "harmonic susceptibility" $\chi_{k}=A_{k} / H_{0}$.

2. Experimental setup. The main problem when studying the ferrofluid nonlinear response is the creation of a strong alternating field. To avoid the sample heating, the field generating and measuring systems are spaced apart and separated by a thermostating screen. The magnetic field is created by a solenoid with an active resistance of $1.3 \mathrm{Ohms}$. At an amplitude of the supply voltage of $10 \mathrm{~V}$, the field amplitude is $\sim 10 \mathrm{kA} / \mathrm{m}$ with a power dissipation of $\sim 100 \mathrm{~W}$. The solenoid is placed in the gap between two coaxial polypropylene pipes through which the cooling liquid is pumped. The measuring system consists of two test-tubes of $9 \mathrm{~mm}$ in diameter and $170 \mathrm{~mm}$ in length, with the measuring coils wound around them. When one of the test tubes is filled with a ferrofluid, a signal proportional to the fluid magnetization appears at the system output. The signal from the empty coil is used to measure the field amplitude. Both signals are applied to the input of the two-channel lock-in amplifier Anfatec eLockIn 203, which measures the amplitudes and phases of the input signals and of their harmonics. The whole system was tested by measuring the mutual induction coefficient. In the frequency range from $40 \mathrm{~Hz}$ to $60 \mathrm{kHz}$ it turned out to be constant with an accuracy of $1 \%$. The main measurements were made at frequencies varying from 4 to $111 \mathrm{~Hz}$.

3. Ferrofluid samples. We investigate two groups of ferrofluids (FF1 and FF2) of the same type (magnetite + kerosene + oleic acid) but with different disperse compositions (i.e. two groups have different size distributions $f(x)$ ). Each group contains four samples differing only in particle concentration. In the group FF $i$, where $i=1$ or 2 , the sample FFi.1 is the least concentrated and in each subsequent sample (FFi.2, FFi.3 and FFi.4) the concentration increases twofold. $f(x)$ for each group is approximated by the $\Gamma$-distribution

$$
f(x)=\frac{x^{\alpha}}{x_{0}^{\alpha+1}} \frac{\exp \left(-x / x_{0}\right)}{\Gamma(\alpha+1)} .
$$

The distribution parameters $\alpha$ and $x_{0}$ were determined by a magneto-granulometric analysis, as described in [9]. Their values along with the relative distribution

Table 1. Results of the magneto-granulometric analysis.

\begin{tabular}{ccccccc}
\hline \hline Sample & $\alpha$ & $x_{0},[\mathrm{~nm}]$ & $\delta$ & $\langle x\rangle,[\mathrm{nm}]$ & $\varphi, \%$ & $M_{\infty},[\mathrm{kA} / \mathrm{m}]$ \\
\hline FF1.3 & 11.06 & 0.84 & 0.29 & 10.2 & 25 & 41.1 \\
FF2.2 & 1.21 & 3.14 & 0.67 & 6.9 & 10 & 13.3 \\
\hline \hline
\end{tabular}


Nonlinear response of a concentrated ferrofluid to a low-frequency magnetic field

Table 2. Initial static susceptibilities of ferrofluid samples at $T=-10^{\circ} \mathrm{C}$.

\begin{tabular}{rcccccccc}
\hline \hline Sample & FF1.1 & FF1.2 & FF1.3 & FF1.4 & FF2.1 & FF2.2 & FF2.3 & FF2.4 \\
$\chi$ & 1.53 & \multirow{2}{*}{3.54} & 7.75 & \multirow{2}{*}{18.6} & 2.83 & 5.63 & 12.6 & 20.4 \\
\hline \hline
\end{tabular}

width $\delta=1 / \sqrt{\alpha+1}$, mean magnetic diameter $\langle x\rangle$, particle hydrodynamic volume fraction $\varphi$ and the saturation magnetization $M_{\infty}$ for two different samples are given in Table 1. It is seen from the data that the sample group FF2 has a wider size distribution than the group FF1. Table 2 additionally gives initial static susceptibilities of all samples at $T=-10^{\circ} \mathrm{C}$.

4. Results. Figs. 1 and 2 show the amplitude dependences of $\chi_{1}$ and $\chi_{3}$ (the susceptibilities of the first two nonzero magnetization harmonics) at $T=$ $-10^{\circ} \mathrm{C}$. For each sample, the harmonic susceptibilities are normalized by the static susceptibilities $\chi$ given in Table 2. Fig. 1 depicts data for the sample group FF1, and Fig. 2 corresponds to the group FF2. It is seen that $\chi_{1}$ monotonically decreases with increasing $H_{0}$, but in the case of $\chi_{3}$, the dependences have a maximum. An increase in concentration (as well as in distribution width $\delta$ ) leads to a faster decline of $\chi_{1}$ and to a shift of the $\chi_{3}$ maximum towards lower amplitudes. In all cases, $\chi_{3}$ is several times smaller than the corresponding $\chi_{1}$. The error of the theoretical predictions for FF1, as a rule, does not exceed 10\%. The exception is the most concentrated sample FF1.4, for which $\chi_{3}$ is much larger than that predicted by MMFT2. For FF2, the accuracy of the theoretical predictions is

$(a)$

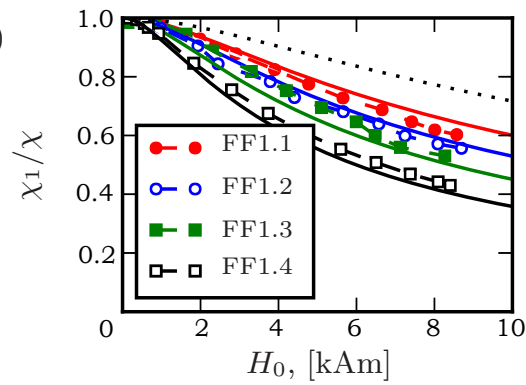

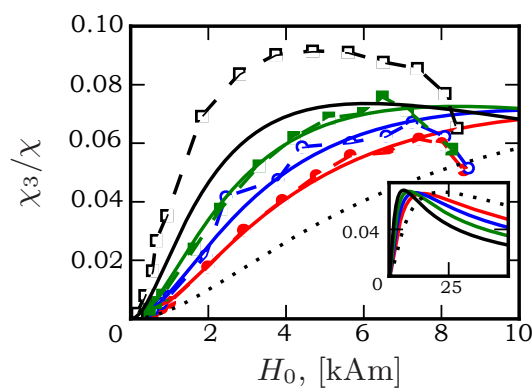

Fig. 1. Normalized susceptibilities of the first $(a)$ and third $(b)$ magnetization harmonics vs. field amplitude for FF1, $T=-10^{\circ} \mathrm{C}$. Solid lines - MMFT2, symbols experimental data (measured at $111 \mathrm{~Hz}$ ), dotted line - Langevin theory. Inset in $(b)$ : theoretical results in a wider amplitude range.

$(a)$

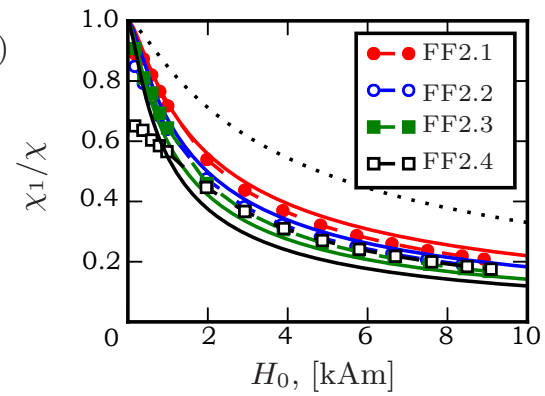

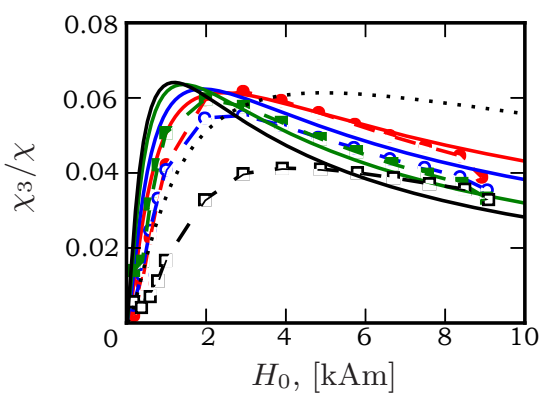

(b)

Fig. 2. The same as in Fig. 1 but for FF2. Data for FF2.1 and FF2.2 are measured at $12.33 \mathrm{~Hz}$, for FF2.3 and FF2.4 at $4.11 \mathrm{~Hz}$. 


\section{A.A. Kuznetsov, A.V. Lebedev, A.F. Pshenichnikov}

(a)

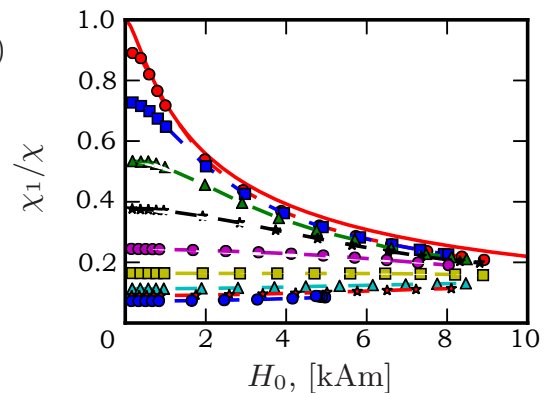

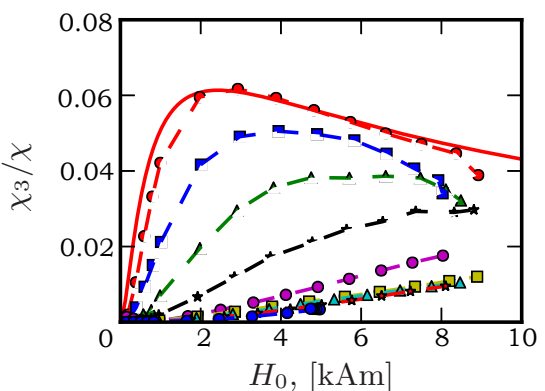

Fig. 3. Harmonics's susceptibilities vs. field amplitude for the sample FF2.1. Solid line - MMFT2, symbols - experimental results at different frequencies: (from top to bottom) $\omega / 2 \pi=12.33,37,111,333,10^{3}, 3 \cdot 10^{3}, 9 \cdot 10^{3}, 27 \cdot 10^{3}$ and $81 \cdot 10^{3} \mathrm{~Hz}$.

somewhat worse-even for dilute samples there is a noticeable discrepancy between the theoretical and the experimental values of $\chi_{3}$ at $H_{0}<2 \mathrm{kA} / \mathrm{m}$ (points lie below curves). Moreover, for the most concentrated samples the first harmonic susceptibility does not coincide with the static ferrofluid susceptibility at small amplitudes $\left(\chi_{1} / \chi<1\right.$ at $\left.H_{0} \rightarrow 0\right)$. Fig. 3 shows the experimental data for FF2.1 measured at different frequencies. With increasing frequency (and hence with increasing $\omega \tau)$, the normalized harmonic susceptibilities decrease and the decrease is stronger in the weak-field limit.

So, it is suggested that the poor agreement between the MMFT2 and FF2 data in Fig. 2 is due to the fact that even at low frequencies $4-12 \mathrm{~Hz}$ the condition of quasi-equilibrium is not fulfilled for this group of samples, i.e. the dimensionless parameter $\omega \tau$ cannot be considered infinitesimal for them. This can potentially explain why in Fig. 2b the data points for FF2.4 lie bellow the experimental results for less concentrated samples at $H_{0}<2 \mathrm{kA} / \mathrm{m}$, whereas MMFT2 predicts the opposite behavior. It is known that interparticle correlations in concentrated ferrofluids lead to a slowing down of the relaxation processes and result in larger relaxation times $\tau$ [6]. It means that at the same field frequency the parameter $\omega \tau$ in concentrated samples is larger than in dilute ones. Therefore, deviations from the quasi-equilibrium regime in concentrated samples should be more pronounced.

5. Conclusion. It is shown that both interparticle interactions and polydispersity have a significant impact on the ferrofluid behavior in a strong alternating magnetic field. Under quasi-static conditions and for moderately concentrated samples $(\varphi<30 \%)$, this impact can be satisfactorily described by MMFT2. However, in order to predict the fluid response at frequencies $\geq 10^{4} \mathrm{~Hz}$, which are typical for some applications [7], an additional account of the relaxation processes (i.e. Brownian and Néel relaxation of magnetic moments) is required.

Acknowledgements. The research is supported by the Russian Foundation for Basic Research under grant no. 16-01-00517.

\section{References}

[1] K.I. Morozov, A.F. Pshenichnikov, Y.L. Raikher, and M.I. Shliomis. Magnetic properties of ferrocolloids: The effect of interparticle interactions. J. Magn. Magn. Mater., vol. 65 (1987), no. 2-3, pp. 269-272.

[2] A.O. Ivanov and O.B. Kuznetsova. Magnetic properties of dense ferrofluids: an influence of interparticle correlations. Phys. Rev. E, vol. 64 (2001), no. 4 , p. 041405. 
Nonlinear response of a concentrated ferrofluid to a low-frequency magnetic field

[3] B. Huke AND M. LüCKE. Magnetic properties of colloidal suspensions of interacting magnetic particles. Rep. Prog. Phys., vol. 67 (2004), no. 10, p. 1731.

[4] A.F. Pshenichnikov And A.V. Lebedev. Magnetic susceptibility of concentrated ferrocolloids. Colloid J., vol. 67 (2005), no. 2, pp. 189-200.

[5] A.O. Ivanov, et al. Magnetic properties of polydisperse ferrofluids: A critical comparison between experiment, theory, and computer simulation. Phys. Rev. E, vol. 75 (2007), no. 6, p. 061405.

[6] A.O. Ivanov, V.S. Zverev, and S.S. Kantorovich. Revealing the signature of dipolar interactions in dynamic spectra of polydisperse magnetic nanoparticles. Soft matter, vol. 12 (2016), no. 15, pp. 3507-3513.

[7] D. Hensley, et al. Combining magnetic particle imaging and magnetic fluid hyperthermia in a theranostic platform. Phys. Med. Biol., vol. 62 (2017), no. 9, p. 3483 .

[8] B.H. Erné, K. Butter, B.W.M. Kuipers, and G.J. Vroege. Rotational diffusion in iron ferrofluids. Langmuir, vol. 19 (2003), no. 20, pp. 8218-8225.

[9] A.F. Pshenichnikov, A.V. Lebedev, A.V. Radionov, and D. Efremov. A magnetic fluid for operation in strong gradient fields. Colloid J., vol. 77 (2015), no. 2, pp. 196-201.

Received 27.12.2017 